\title{
Getting better - health profession knowledge is key to improving deceased donation practices in South Africa
}

The early identification and referral of potential organ donors is essential to the organ donation process. Without recognition of the potential for donation by the treating clinical team, the process simply does not start. In their well-structured article, Prins and Human ${ }^{[1]}$ have shown gaps in knowledge, and the need for education on the key steps in the organ donor pathway in South Africa (SA).

While this study focuses on the intensive care unit (ICU) nurse, it could easily be extended to include the treating physician. End-of-life care is provided by a team interacting with the patient and family. The barriers highlighted by Prins and Human ${ }^{[1]}$ (perceived extra work that taking a donor family through the consent process entails, lack of policy and guidance) are very real, and it is important that clinicians, nurses, hospital managers and transplant teams work within a system that ensures that the exploration of organ and tissue donation is a standard of care offered routinely at the end of life. This starts with assessing knowledge. As clinicians it is important to recognize that it is paternalistic of us to not give a patient and their family the option to support organ and tissue donation at the end of life whenever it is possible.

A striking feature of the study is that $97 \%$ of respondents failed to identify potential donors, and even after the educational intervention, $45 \%$ missed potential donors. Countries with high organ donation rates have accepted triggers for organ donor assessment and referral. This ensures that the assessment of donation potential is made by transplant teams, who are best positioned to make such an assessment, and that timeous and informed discussions are held in an often emotional and stressful time for families and clinicians alike. The window for a successful transplant operation can be mere hours for patients on the waiting list. In SA, hearts, lungs and livers are allocated to the sickest patients first, using objective scoring systems. The general ICU clinician or nurse is not aware of these urgent calls and therefore not able to make a call on the suitability of marginal organs for these patients.

In the USA, all hospitals are required to notify every death to an accredited organ procurement organisation. Their federal funding is dependent on it. ${ }^{[2]}$ This helps to ensure that any opportunity for tissue donation is not lost. While organ donation may only be possible in certain circumstances, the potential for tissue donation is greater, since tissues can be recovered for some time after death certification. In the setting of National Health Insurance in SA, it will be important that institutions are required to have systems in place to offer optimum endof-life care that includes mechanisms to monitor and improve our organ donation rates. This should include routine assessment and approach to families about organ and tissue donation.

In the UK, there are minimum notification criteria to identify all potential organ donors. The National Institute of Health and Clinical Excellence recommends that hospital staff initiate discussions with a specialist nurse for organ donation (SN-OD) when one of the following criteria is met: $(i)$ defined clinical trigger factors are met in patients who have had a catastrophic brain injury, namely the absence of one or more cranial nerve reflexes, and a Glasgow Coma Scale score $\leq 4$ that is not explained by sedation, and/or a decision has been made to perform brain-stem death tests, whichever is the earlier; or (ii) the intention is to withdraw life-sustaining treatment in patients with a life-threatening or life-limiting condition that will, or is expected to, result in circulatory death. ${ }^{[3]}$

These criteria allow for early discussion with an expert in donation, and for consideration of organ donation after circulatory death. This form of organ donation is not offered in the private sector in SA, but is established practice in state hospitals in the Western Cape ${ }^{[4]}$ In the UK, over one-third of deceased donors are donors after circulatory death.

A recent paper from the Johannesburg group showed that following a structured process, with defined roles, a well-planned family approach can have a large impact in consent rates, improving a $25 \%$ consent rate to $73 \%{ }^{[4]}$ National legislative changes such as presumed consent legislation are often touted as a way to improve organ donation rates. Interestingly, the Spanish system, currently the highest performing in the world, only saw an increase in deceased donation after the formation of a national co-ordinating body and once other quality assurance mechanisms were put in place, which was 10 years after presumed consent legislation was enacted. In Spain, the family is always approached for consent, and the refusal rate is $16.4 \%$ even with presumed consent. ${ }^{[6]}$ The manner, environment and timing of requesting consent are key to success, and best done by a specially trained requestor.

Prins and Human ${ }^{[1]}$ also looked at donor management knowledge, another key step where the ICU can impact on transplant outcomes through optimal preservation (and resuscitation) of organs prior to the organ recovery operation. It is also interesting to note the knowledge gaps shown with regard to brain death certification. Confidence in the diagnosis of death by the treating team and the family is integral to the organ donation process. Education is required to address these concerns on the part of treating teams, as death must, and can always be, certified with complete certainty. There are well-established international guidelines on death determination, and the Critical Care Society of Southern Africa is currently in the process of putting together SA guidance in line with the most recent evidence to ensure that the highest standards are adhered to, and aligned with our legislation.

There are limitations to this study in that it only surveyed 'interested' nursing personnel from the ICUs of five private hospitals. Referrals often come from busy emergency units, especially in the state sector, so training efforts need to include these healthcare professionals, and would need to assess knowledge and attitudes to donation in these units. As with all educational interventions, it is important to recognise that knowledge is dynamic, and was in this case assessed immediately after a training intervention. It would be interesting to know from longterm follow-up how much knowledge was retained, and whether the educational intervention had a tangible impact on practice, as per the Kirkpatrick model to assess educational interventions. Thankfully the authors have conducted this study within a larger ongoing education initiative across 32 hospitals, and are to be congratulated on this programme. Education is an important step twards establishing a culture of donation across our healthcare institutions. 
D Thomson, FCS (SA), Cert Crit Care (SA)

Division of Critical Care, Department of Surgery, Groote Schuur Hospital and University of Cape Town

thomson.david@gmail.com

South Afr J Crit Care 2019;35(2):39-40. https://doi.org/10.7196/SAJCC.2019.v35i2.414

\footnotetext{
1. Prins LJ, Human L. Early identification and referral of organ donors in five private hospitals: A survey to determine the knowledge and views of critical care professional nurses pre and
}

post a PowerPoint training intervention. South Afr J Crit Care 2019;35(2):50-57. https://doi. org/10.7196/SAJCC.2019.v35i2.370

2. Centers for Medicare and Medicaid Services. Medicare and Medicaid programmes: Conditions for coverage for organ procurement organisations (OPOs). Federal Register 2006;71(104):30981.

3. National Health Service. Timely identification and referral of potential organ donors. Leeds: NHS, 2012. https://nhsbtdbe.blob.core.windows.net/umbraco-assets-corp/1337/timely-identificationand-referral-of-potential-organ-donors-nhsbt.pdf (accessed 16 June 2019).

4. Du Toit T, Manning K, Thomson D, et al. Kidney transplantation utilising donors after circulator death - the first report from the African continent. Transplantation 2018;102:S493-S94. https:/ doi.org/10.1097/01.tp.0000543311.81537.58

5. De Jager M, Wilmans C, Fabian J, Botha J, Etheredge H. Increasing deceased organ donor numbers in Johannesburg, South Africa: 18-month results of the Wits Transplant Procurement Model. S Afr Med J 2019;109(9):626-631. https:doi.org/10.7196/SAMJ.2019.v109i9.14313

6. Rodriguez-Arias D, Wright L, Paredes D. Success factors and ethical challenges of the Spanish Model of organ donation. Lancet 2010;376(9746):1109-1112. https://doi.org/10.1016/s0140 6736(10)61342-6 